\title{
UWB Based Integrated Communication and Positioning System for Multi-UAVs Close Formation
}

\author{
Shuqiang $\mathrm{Cao}^{\mathrm{a}}$, Yongbin $\mathrm{Zhou}^{\mathrm{b}}$, Dong Yin ${ }^{\mathrm{c}}$ and Jun Laid \\ College of Artificial Intelligence, National University of Defense Technology, \\ Changsha 410000, China \\ acaoshuqiang01@163.com, ’mz323@126.com, cyindong@nudt.edu.cn, dlaijun@nudt.edu.cn
}

Keywords: Close formation, UWB, Positioning, Communication

\begin{abstract}
Multi-UAVs close formation is the hot spot of current research. UWB (ultra-wide band) is very feasible to be applied to UAV relative positioning. In this paper, we propose to apply UWB technology to UAV relative positioning for multiple UAV close formations and consider combining UWB positioning with communication to fuse the state information of UAVs in close formation. The integrated communication and positioning system is constructed and its performance is evaluated by indoor test, outdoor comparison and flight experiment. Experimental results shows the effective
\end{abstract}

\section{Introduction}

Multi-UAV formation, which can accomplish the task cooperatively and improve the success probability of mission, is arousing the research upsurge. The close formation of UAVs has the characteristics of light weight, high aspect ratio, good aerodynamic performance and high structural strength. [2] Therefore it is the hot spot of recent research. Compared with single UAV flight, multi-UAV compact formation is more complex and needs to break through a series of technical difficulties. Relative positioning between UAVs is the key problem of close formation.

The ultra-wide-band (UWB) signal uses narrow pulse with very short duration, which has good resolution for time and space. The positioning accuracy can reach centimeter level if take no other errors into consider. Compared with the traditional narrow band system, it has the advantages of strong penetration, low power consumption, good multipath resistance, high security, low complexity of the system and accurate positioning accuracy. Fontana et al's research shows that UWB can even achieve 2 centimeters accuracy when locating static targets, while the accuracy of dynamic target positioning can also be controlled within 30 centimeters. [7,8,9] Therefore it is very feasible to apply UWB technology to UAV relative positioning.

In this paper, we propose to apply UWB technology to relative positioning for multi-UAV close formation and combine UWB location with communication to fuse the state information of UAVs. An UWB based integrated communication and positioning system is constructed to obtain high positioning accuracy in UAV formation.

\section{System Principle}

There are several kinds of UAV formation at present. In this paper, the most popular "leader-follow" formation method is chosen to study. Relative positioning in formation is to obtain the relative distance D, yaw angle $\varphi$ and pitch angle $\theta$ between lead UAV and follow UAV.

\subsection{Relative Positioning}

Distance estimation based on TOA is a traditional and most effective method and the use of two-way ranging (TWR) algorithm does not require data synchronization between nodes. [10] In this paper, DS-TWR method is used to calculate the time of signal propagation and complete the distance measurement. [11] 
According to the principle of TOA positioning, three Anchor nodes are designed to locate the Tag node. Based on the distances, three equations are established. The height information of UAV is introduced to simplify the equations and get the only location coordinates.

There are many algorithms to solve the distance equations, such as direct calculation, least square method, Fang algorithm, [12] Chan algorithm, [13] Taylor algorithm [14] and so on. In the case of noise interference, the accuracy of direct calculation is low and there used to be no solution. The Chan algorithm is a two-order weighted least square method which has computational complexity. The Taylor algorithm needs an initial value close to the real value otherwise it may lead to a local optimal solution. The least square method is a mathematical optimization technique that minimizes the squared sum of errors to find the best function matching of the data. Considering the accuracy and computational complexity, the least square method is most appropriate in this paper.

\subsection{Communication Mechanism}

There are diverse communication requirements for the UAV system. From the perspective of tight formation, the communication system is to complete the interaction between UAVs so that each UAV in the formation "knows" the position and status of other UAVs. Then the UAV can constantly modify the position of the aircraft in the formation and maintain the topology of formation.

The information of UAVs can be sent to the ground station and the status information of all UAVs are broadcasted by the ground station so as to realize the information exchange. But this communication link is too long that the data delay is large and the data reliability and security of multiple transmission will be reduced. So inter-UAV communication mechanism is more suitable for close formation.

\section{System Design}

In the "Lead - Follow" formation, the lead UAV will fly in accordance with the set route and the follow UAV automatically follows the lead UAV. So lead UAV needs to install GPS and UWB nodes to complete the positioning, flight control module to complete the, inter-UAV communication equipment to complete communication. In addition to these modules, the follow UAV needs processor module to realize the formation control algorithm. The structure of the whole system is shown in Fig.1.

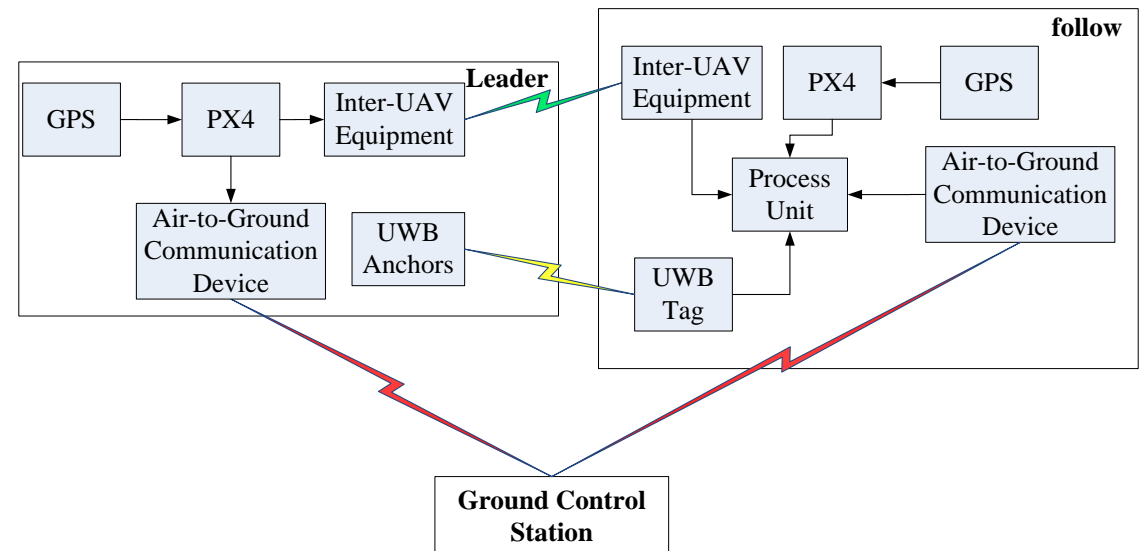

Figure 1. Structure of System

It is quite important for the computer to process, judge and fuse GPS data and UWB data which needs a closed control loop. The integrated communication and positioning system mainly comprises a GPS module, a UWB module, a data synchronization / fusion module and a driving module to form a closed control loop. The working flow diagram of the system is shown in Fig.2. 


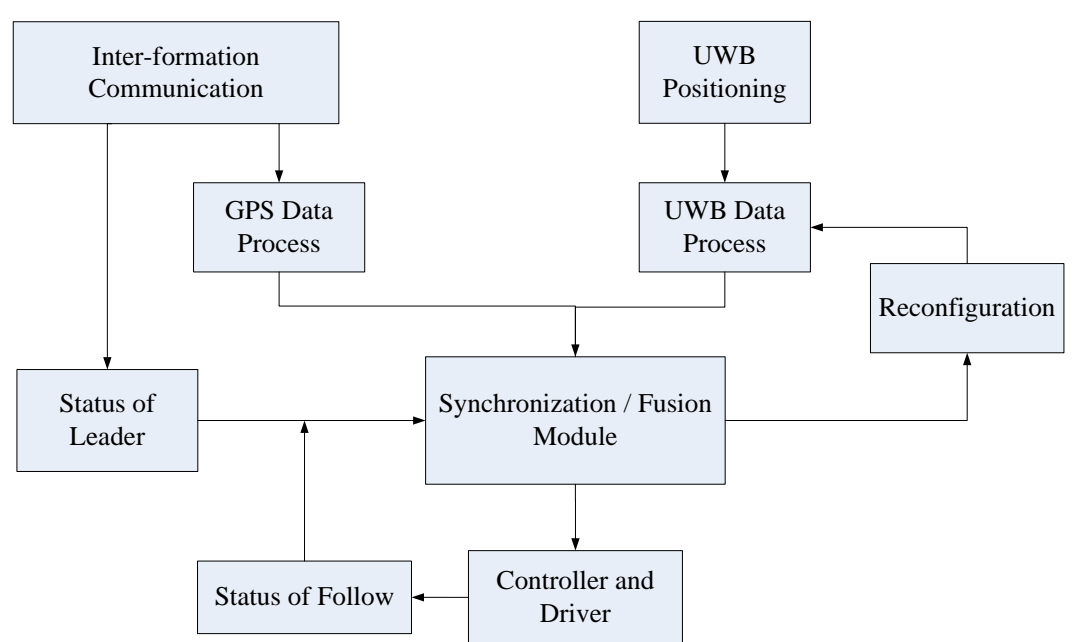

Figure 2. Working Flow of System

Considering that the effective distance of UWB module is limited, the GPS is needed to guide the follow UAV to approach the lead UAV. Once the relative distance reaches within the effective working distance of UWB, UWB data can be timely introduced to realize the close formation. The control process of formation is shown in Fig.3.

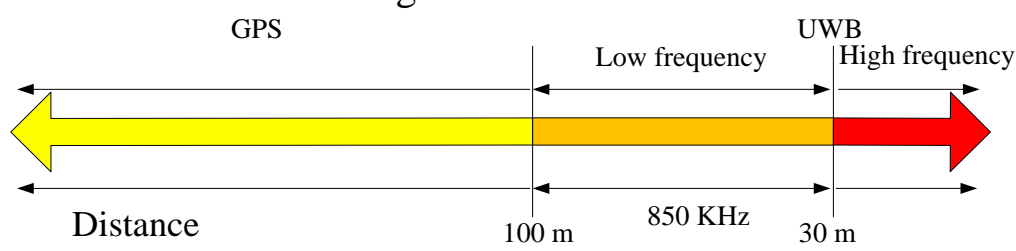

Figure 3. Diagram of Formation Control Process

In process of formation control, the processor detects whether there is stable UWB positioning data while the follow UAV gets close to lead UAV. When the UWB module can stably operate in the mode with the data rate of $850 \mathrm{KHz}$, the follow UAV is controlled according to the low frequency UWB positioning data. As the distance decreases, the formation can be realized by using the high frequency UWB positioning data when the UWB module can work steadily in the mode with data rate of $6.8 \mathrm{MHz}$. If the distance between UAVs increases, causing the UWB data not stable, it is necessary to switch back to the GPS positioning in time to ensure the relative position can be got in the whole process.

\section{Relative Positioning}

In the system design scheme, the GPS data processing module, the UWB data processing module and the data fusion processing module are the core modules to realize the relative positioning. The GPS data processing module mainly plays a role in the flight guidance phase while the UWB module mainly plays a role in close formation.

\subsection{GPS Process Module}

The GPS processing module plays a role in calculating the relative distance and angles based on the GPS data of lead UAV and follow UAV. The GPS data gets filtered firstly to eliminate rough position error and reduce noise interference. Then the longitude, latitude and height are converted into space coordinates for space position solving. Its composition is shown in Fig.4. 


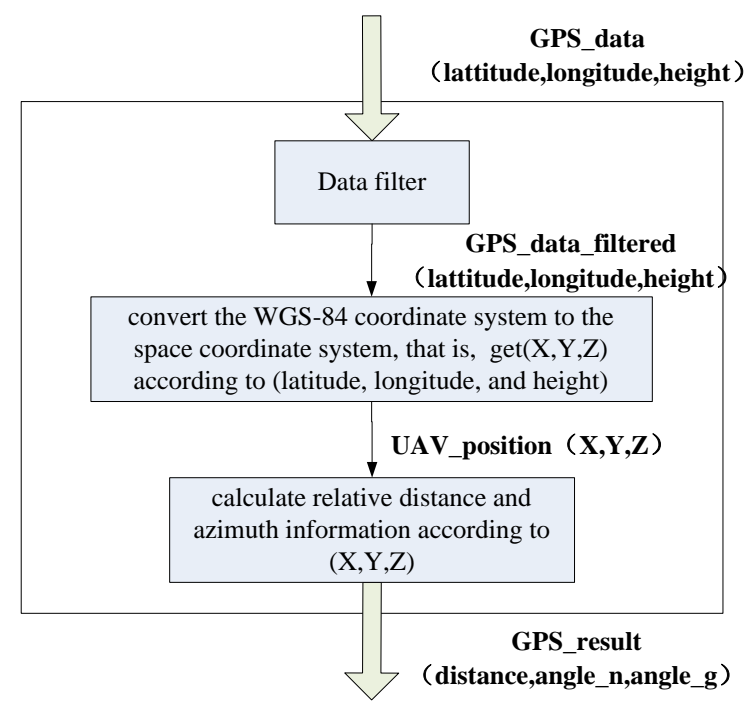

Figure 4. Diagram of GPS Data Process

\subsection{UWB Process Module}

The function of UWB data processing module is to calculate the relative position between UAVs according to the measurement information. To achieve the accuracy of centimeter level, it is necessary to make more complicated processing for UWB data. On the one hand, we need to filter the UWB ranging data and eliminate gross errors. On the other hand, the flight states of UAVs are introduced to predict the relative position, estimate the position change of UAV and verify the relative positioning results. The process schedule is shown in Fig.5.

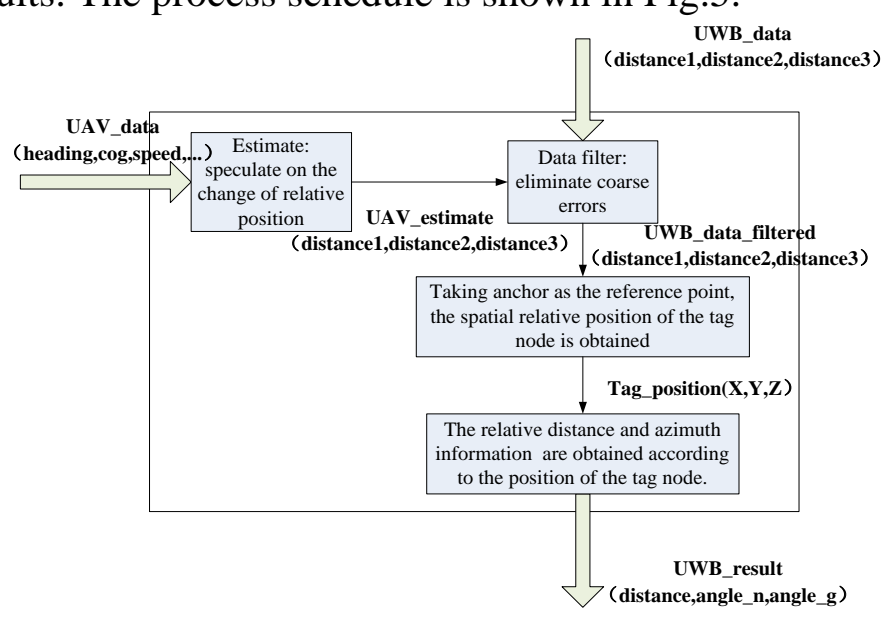

Figure 5. Diagram of UWB Data Process

A coordinate system is established in order to facilitate calculation and analysis, where the origin is set on the centroid of the lead UAV, the $X$ axis is the forward direction of head of the lead UAV, the $\mathrm{Y}$ axis is the forward of right wing, and the $\mathrm{Z}$ axis direction towards the bottom of the fuselage which is determined by the right-hand screw rule.

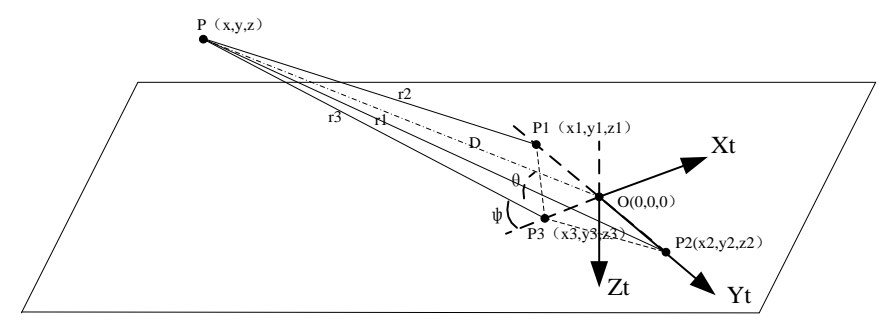

Figure 6. Simplified Diagram of Coordinate System

Supposed Anchors are $\mathrm{P}_{i}\left(x_{i}, y_{i}, z_{i}\right), \mathrm{i}=1,2,3$, the Tag node is $\mathrm{P}\left(x_{t}, y_{t}, z_{t}\right)$ and the distance between $P_{i}$ and ${ }^{\mathrm{P}}$ is $r_{i}$. Thus 
where $z_{t}, x_{i}, y_{i}, z_{i}(i=1,2,3)$ are all known.

$$
\left(x_{i}-x_{t}\right)^{2}+\left(y_{i}-y_{t}\right)^{2}+\left(z_{i}-z_{t}\right)^{2}=r_{i}^{2} .
$$

According to the least square method, the matrix form can be obtained from the equations.

Where

$$
H \times x=b \text {. }
$$

$$
\begin{gathered}
H=\left[\begin{array}{cc}
x_{2}-x_{1} & y_{2}-y_{1} \\
x_{3}-x_{1} & y_{3}-x_{1}
\end{array}\right], \quad x=\left[\begin{array}{c}
x_{t} \\
y_{t}
\end{array}\right], \\
x_{2}^{2}+y_{2}^{2}-r_{2}^{2}-\left(x_{1}^{2}+y_{1}^{2}-r_{1}^{2}\right) \\
b=\frac{1}{2}\left[\begin{array}{c}
x_{3}^{2}+y_{3}^{2}-r_{3}^{2}+z_{3}{ }^{2}-2 z_{3}{ }^{\circ} z_{t}-\left(x_{1}^{2}+y_{1}^{2}-r_{1}^{2}\right)
\end{array}\right] .
\end{gathered}
$$

Then the minimum variance solution of $\left(x_{t}, y_{t}\right)$ is

$$
\left[\begin{array}{l}
x_{t} \\
y_{t}
\end{array}\right]=\left(H^{T} H\right)^{-1} H^{T} b
$$

In order to make the result of UWB positioning well utilized in the formation control algorithm, it is necessary to convert the coordinates from the airframe coordinate system to the airborne geographical coordinate system.

Assuming $C_{g}$ is the transformation matrix of the body coordinate system to the airborne geographic coordinate system, $[15] \mathrm{P}(x, y, z)$ in the airborne geographic coordinate system is:

$$
\left[\begin{array}{l}
x \\
y \\
z
\end{array}\right]=C_{g} \times\left[\begin{array}{l}
x_{t} \\
y_{t} \\
z_{t}
\end{array}\right] .
$$

The relative position relationship can be calculated as follows.

$$
\begin{aligned}
& \text { distance }=\sqrt{x^{2}+y^{2}} . \\
& \text { angle }_{n}=\tan ^{-1} \frac{y}{x} .
\end{aligned}
$$

\subsection{Data Fusing Module}

GPS positioning and UWB positioning are combined to realize accurate positioning and formation control especially when GPS method and UWB method need to be switched. The data fusing module is designed to realize this function. The state information of UAVs is utilized to determine which method is more appropriate under the current situation. Data fusing module makes data comparison and selects positioning method in time so that there is no upheaval in relative positioning results when the positioning mode is switched. The essential purpose is to make sure UAVs can fly stably in the formation. The procedure is shown in Fig.7.

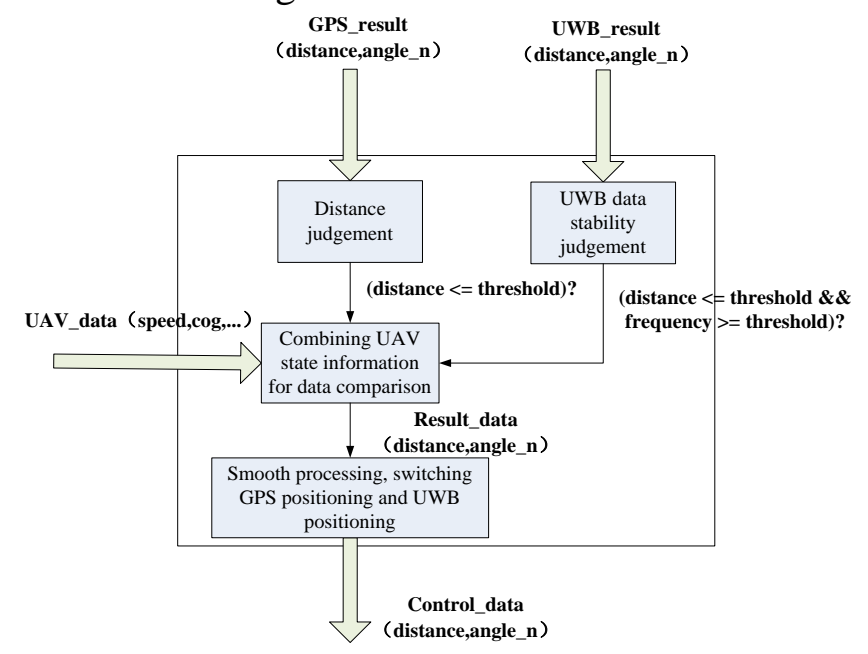

Figure 7. Diagram of Data Fusing Process

Whether UWB positioning is stable is firstly judged when GPS data and UWB data are fused. If the UWB module cannot work stably, the positioning results of GPS are directly output as relative positioning result. When the UWB module is stable, the results of GPS positioning and the result of UWB positioning are compared. If the results of the two approaches, it is believed that UWB 
positioning is accurate. If the two differences, then it need to determine which positioning results is more credible according to the state of UAVs, such as light speed, track angle, and so on.

\section{Inter-UAV Communication}

In order to calculate the relative positioning between UAV, it is also necessary to transmit the UAV status data packet through inter-UAV communication. To establish the communication link between UAVs, it must follow the communication protocol and construct the corresponding data packet.

The MAVLink (Micro Air Vehicle Link) protocol is a higher-level open source communication protocol based on serial communication, which is the most commonly used communication protocol between UAVs and ground station. The MAVLink protocol is used in this paper but communication data packets are simplified to ensure smooth communication link between UAVs and reduce the distortion and hysteresis. The structure of communication data packet is shown in Fig.8.

\begin{tabular}{|}
\begin{tabular}{|c|c|c|c|c|c|c|c|c|c|c|}
\hline Head & Seq & Len & \multicolumn{3}{|c|}{ Data } & CRC \\
\hline 1B & 3B & 1B & \multicolumn{3}{|c|}{$40 \mathrm{~B}$} & 2B \\
\hline Lat & Lon & High & Roll & Pitch & Yaw & Cog & Vn & Ve & Vd \\
\hline 4B & 4B & 4B & 4B & 4B & 4B & 4B & $4 B$ & $4 B$ & $4 B$ \\
\hline
\end{tabular}
\end{tabular}

Figure 8. Structure of Communication Data Packet

The status of the lead UAV, including position, attitude velocity and so on, is sent to the follow UAV by using data packets with only 47 bytes. The capacity of the occupied channel is small so that it can meet the demand of high frequency communication.

\section{Experiment and Analysis}

DWM1000 is chosen to be the positioning module in this system. The module is based on the DW1000 chip, developed by DecaWave Company, which is an UWB wireless transceiver module compatible with the IEEE 802.15.4-2011 protocol.

As is shown in Fig.9, three DWM1000 modules are fixed on leader UAV as anchor nodes; a tag node, subjected to ranging with three anchors respectively, is fixed on follow UAV.
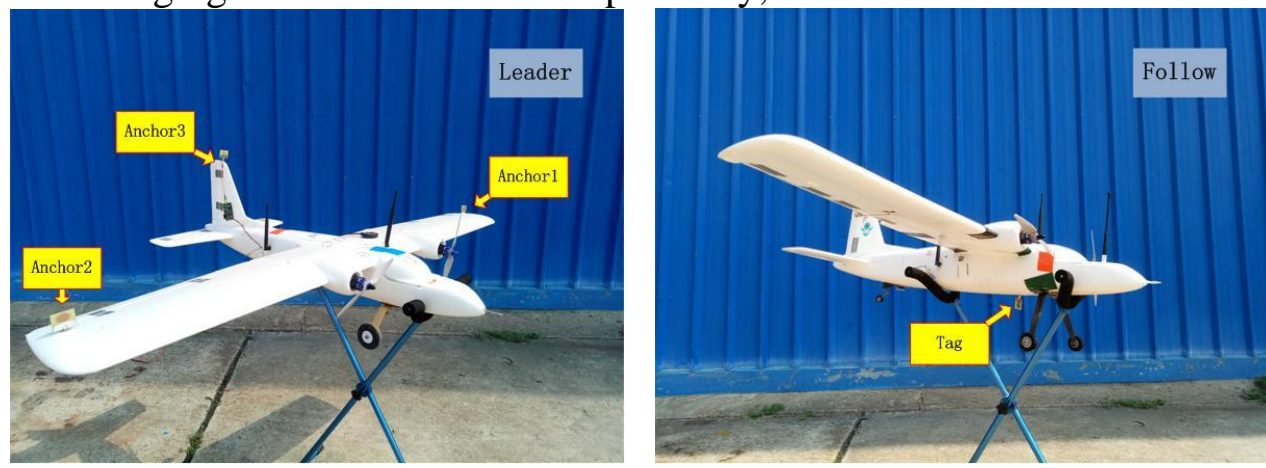

Figure 9. Actual Installation Situation

\section{Indoor Test}

The purpose of the indoor test is twofold : one is to test the ranging performance of the UWB module, including the zero deviation, the effective distance precision and so on. The second is to verify whether the algorithm is correct and whether the accuracy meets the need of relative positioning.

VICON system is chosen to provide reference for positioning results in this paper,. VICON is an optical motion capture system produced by OML Company in UK. Its positioning accuracy can reach millimeter level. In VICON system, the coordinate of each UWB module is measured by maker ball. 
A coordinate system is established by Anchor nodes which coincides with VICON coordinate system. The coordinate of Tag node is calculated by UWB positioning while VICON system provides a positioing reference. The results are shown in Fig. 10.

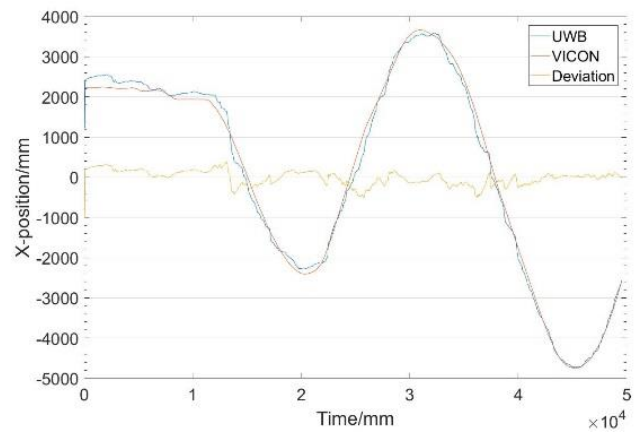

a) X label

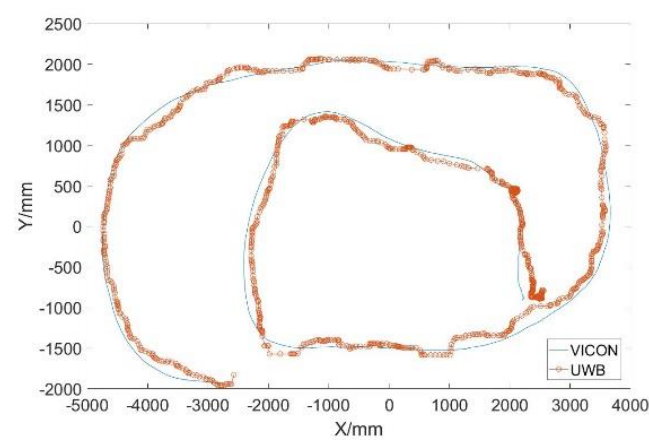

c) Route Comparison

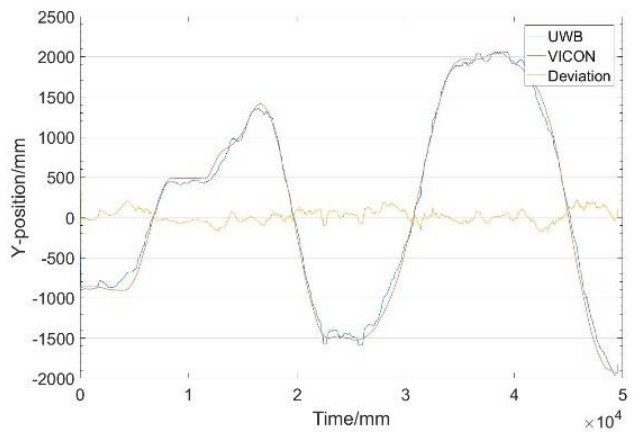

b) Y label

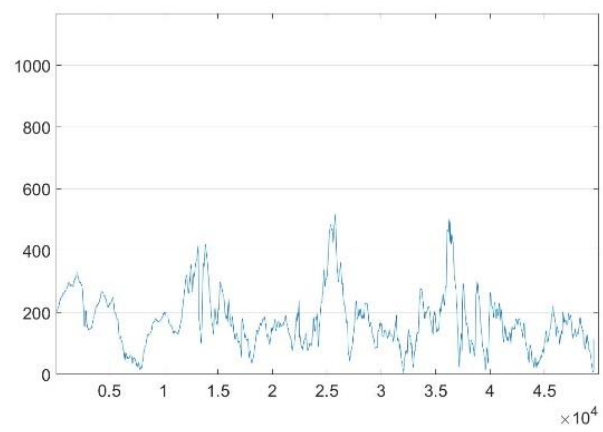

d) Positioning Error

Figure 10. Results of Comparison Between UWB And VICON System

As can be seen from the results of the comparison, the accuracy of UWB positioning is about 20 $\mathrm{cm}$ in case of ranging error and noise interference. But the accuracy may be increased to about $40 \mathrm{~cm}$ in case, for example a turn, where the DOP is low. In general, this positioning accuracy can satisfy the requirements of UAV positioning.

\section{Outdoor Comparison}

There are two purposes for outdoor testing. One is to determine the effective working distance of UWB module. The other is to verify whether UWB location is feasible by comparing UWB positioning results with the GPS.

During the test, the UAV will be stationary for a period of time, then the position of the UAV will be changed. This will facilitate the observation of UWB and GPS positioning results. The distance between UAVs can be increased continuously until the UWB module cannot work so that the effective working distance of UWB can be determined. The result of outdoor comparison is figured in Fig.11.

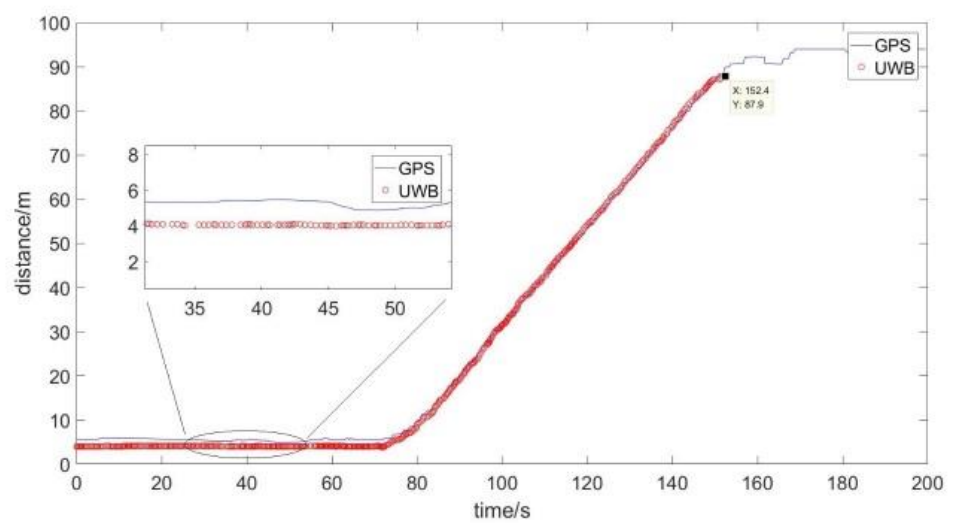

Figure 11. Result of Outdoor Comparison 
It can be seen that the effective working distance of the UWB module is about $87.9 \mathrm{~m}$ which can meet the demand of relative positioning in multi-UAVs compact formation. The positioning accuracy of UWB is obviously higher than that of GPSs under static condition. Although it is not possible to distinguish the performance of UWB and GPS in moving case, it can be confirmed that UWB can completely meet the requirements of relative positioning.

\section{Flight Experiment}

On the basis of the test experiments, it is considered that UWB positioning can be applied to the close formation of UAV. After the simulation experiment and the hardware-in-the-loop simulation, the flight experiment is carried out and the result of the experiment is shown in Fig.12.

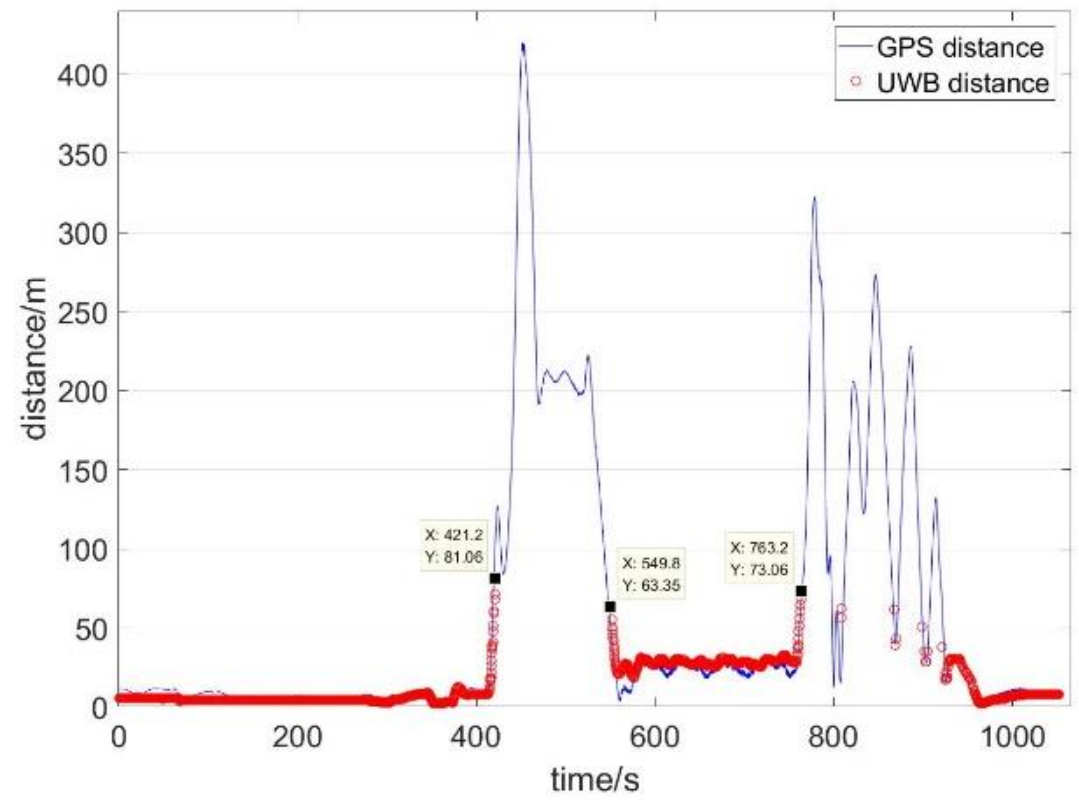

Figure 12. Result of Flight Experiment

The flight experiment consists of a preparation stage, a take-off stage, a GPS guide stage, a formation stage and a landing stage. In the preparation stage, the UWB module can stably work and the positioning result is close to that of GPS. In the take-off stage, the distance between UAVs increases rapidly even beyond the effective range of UWB and the UAVs are positioned only by GPS. In the guide stage, the relative distance gradually decreases and the UWB module returns to work. In the formation stage, the formation control is carried out according to the result of UWB positioning. In the landing phase, the UWB module can hardly measure the distance because of the great altitude difference between UAVs. When both UAVs land, the UWB recovers to range.

Although there are positioning errors in the UWB module and the GPS module, the experimental results are sufficient to demonstrate that UWB techniques are fully feasible for UAV positioning.

\section{Results Analysis}

It can be seen from the experimental results that the effective working distance of the UWB is about $80 \mathrm{~m}$ and the positioning accuracy can reach the decimeter level.

The main factors affecting positioning accuracy are as follows. Firstly, the relative motion of UAVs leads to the difference of bidirectional channel in ranging process. The distance measurement error based on TWR is obviously increased, which leads to the error of the obtained Tag node coordinates. The least square method can only find an optimal solution and cannot eliminate this error in the process. Secondly, distance ranging between nodes is carried out by polling and the nodes are in motion. Therefore, the relative position changes when different Anchor nodes range to Tag node, which will inevitably lead to the positioning error. As a result of my experiment, polling interval is about $24 \mathrm{~ms}$ and the relative velocity between UAVs can even reach to $10 \mathrm{~m} / \mathrm{s}$, which leads to an error of $24 \mathrm{~cm}$. Thirdly, state information and positioning data cannot be fully synchronized. Each time the 
positioning data is read and processed, there will be a delay of $40 \sim 50 \mathrm{~ms}$ when the status information is read. Therefore, there will be a large lag in filtering the location data.

For the above factors affecting accuracy, some optimization schemes can be utilized on the system design and data processing. Firstly, the communication link can be optimized by introducing the scheduling algorithm of the data packet to reduce the time interval between the state information and the positioning data. Secondly, the motion state equation of each node can be established according to the topology of nodes and the motion state of the UAVs. Thus the accuracy of ranging can be improved by filtering and compensating the ranging data directly. Last but not least, Taylor algorithm and other positioning algorithms can be combined in the process of location solving to reduce errors in coordinate solution.

\section{Summary}

In this paper, UWB is applied in relative positioning in multi-UAVs close formation and a UWB based integrated communication and positioning system is designed. Through experiments and the actual flight, the system can meet the demand of close formation.

This system is an exploration and verification of application of UWB technology in fixed wing UAV positioning. It is also an innovation of multi-UAVs close formation positioning. The system realizes the fusion of GPS positioning technology and relative positioning technology. It can not only meet the requirements of UAV positioning under wide-area conditions, but also improves the positioning precision of UAV in the local area.

The system can be easily applied to multi UAV tight formation because different Tag nodes can be identified by Anchor nodes. If more optimized positioning algorithms, more accurate filtering techniques and more intelligent control strategies are employed, the system will be more perfect.

\section{References}

[1]. Fan Qiongjian, Yang Zhong, Fang Ting, Shen Chunlin. Research Status of Coordinated Formation Flight Control for Multi-UAVs [J]. Acta Aeronautica et Astronautica Sinica. Vol.4 (2009) No.30, p. 683-691.

[2]. Wang Jinyun, Wei Ruixuan, Liu Yue, Zhou Wei. Close Formation Configuration Control of Cooperative UAV [J]. Flight Dynamics. Vol.26 (2008) No.6, p. 34-37.

[3]. Information on: http://tech.sina.com.cn/d/i/2015-04-16/docicczm-vun 9567644.html.

[4]. Pang Yan, Qiao Jing. Discussion on UWB Wireless Positioning Technology. Telecom Express. Vol.11 (2005).

[5]. Helmut Hlavacs. Cooperative Enhancement of Position Accuracy of Unmanned Aerial Vehicles.

[6]. Chin-Der Wann, Yi-Jing Yeh and Chih-Sheng Hsueh. Hybrid TDOA/AOA Indoor Positioning and Tracking Using Extended Kalman Filters [J]. IEEE.2006, p. 1058-1062.

[7]. R. Fontana, E. Richley, and J. Barney. "Commercialization of an ultra-wide band precision asset location system [J]," in Proc. of the IEEE Conf. on Ultra-Wideband Systems and Technologies. 2003, p. 369 - 373.

[8]. C. Correal, S. Kyperountas, Q. Shi, and M. Welborn. "An UWB relative location system [J]," in Proc. of the IEEE Conf. on Ultra-Wideband Systems and Technologies. 2003, p. $394-397$.

[9]. G. MacGougan, K. O’Keefe, and R. Klukas, "Tightly-coupled GPS/UWB integration [C]," Journal of Navigation, Vol. 63 (2010) No. 1, p. 1-22.

[10]. Yin Zhen, Huang Huizhe, Li Wei, Lu Yi. TOF Accurate Position System Based on SDS-TWR Algorithm. Experiment Technology and Management. Vol. 34 (2017) No. 5, p. 95-98. 
[11]. Information on: https://www.decawave.com/suport:APS013: DW1000 and Two Way Ranging.

[12]. B.T.Fang. Simple solutions for hyperbolic and related position fixes [J]. IEEE Transactions on Aerospace and Electronic Systems, Vol.26 (1990) No.5, p. 748-753.

[13]. Y.T.Chan, K.C.Ho. A Simple and Efficient Estimator for Hyperbolic Location [J]. IEEE Trans. On Signal Processing. Vol.42 (1994) No.8, p. 1905-1915

[14]. Jiang Wenmei, Wang Mei. Pseudorandom Code Correlation-based UWB Muliti-points Cooperation Location Algorithm [J]. Journal of Guilin University of Electronic Technology. Vol.26 (2006) No.1, p. 1-5.

[15]. Chang Jun. Positioning of Airbone Radar Targets in 54 Coordinate [J]. Vol.2 (2003), p. 97-100. 